\title{
Spinopelvic balance: new biomechanical insights with clinical implications for Parkinson's disease
}

\author{
Luciano Bissolotti • Massimiliano Gobbo • \\ Jorge Hugo Villafañe $\cdot$ Stefano Negrini
}

Received: 13 April 2013/Revised: 26 September 2013/Accepted: 9 October 2013/Published online: 27 October 2013

(C) Springer-Verlag Berlin Heidelberg 2013

\begin{abstract}
Purpose The aim of this study was to describe the disease-related sagittal balance changes in relation to the sacropelvic morphology of PD patients with different durations of disease.

Methods Thirty-one consecutive Parkinson's disease patients (26 males, 5 females; age 55-83 years) participated in the cross-sectional study. The clinical assessment included: Hoehn Yahr score; plumb line distance from the spinous process of C7, kyphosis apex, spinous process of L3 and S1. Lumbar lordosis (LL), thoracic kyphosis (TK), spinosacral angle, spinopelvic angle, spinal tilt, pelvic incidence, sacral slope (SS) and pelvic tilt were radiographically assessed.
\end{abstract}

L. Bissolotti $(\bowtie)$

Rehabilitation Service, Fondazione Teresa Camplani-Casa di Cura

Domus Salutis, Brescia, Italy

e-mail: luciano.bissolotti@ancelle.it

L. Bissolotti · M. Gobbo

LARIN, Neuromuscular and Adapted Physical Activity

Laboratory, Brescia, Italy

M. Gobbo

Department of Clinical and Experimental Sciences,

University of Brescia, Brescia, Italy

J. H. Villafañe $\cdot$ S. Negrini

IRCCS Fondazione Don Gnocchi ONLUS, Milan, Italy

S. Negrini

Physical and Rehabilitation Medicine, University of Brescia, Brescia, Italy
Results Radiographic spinopelvic angles appeared normal, but many patients presented variations from normality. In particular, pelvic tilt increased and SS decreased; spinosacral and spinopelvic angles were greatly reduced compared to healthy people, and spinal tilt increased. Unlike TK, LL was well correlated with most of the parameters.

Conclusions Sagittal balance evaluation provides new valuable insights for biomechanical understanding of PD patients. Specific spinal parameters (spinosacral, spinopelvic and spinal tilt angles), and their clinical correlation, as well as pelvic parameters like pelvic tilt and sacral slope, appear particularly interesting for their clinical implications in terms of spinal deformities correction in PD population.

Keywords Sagittal $\cdot$ Spinopelvic $\cdot$ Parkinson's disease $\cdot$ Fall risk

\section{Introduction}

Today, the importance of sagittal plane alignment for the treatment of spinal disorders is widely recognized in the scientific world [1-6]. The understanding of the regulatory principles of sagittal balance is useful to achieve good outcomes when treating different spinal disorders in the field of orthopedic rehabilitation and spine surgery, especially when treating major deformities $[3,7,8]$. Indeed, the influence of sacropelvic balance and morphology has been widely evaluated in the literature concerning adult spinal deformity [12], adolescent idiopathic scoliosis [1, 9], spondylolisthesis [14], and low back pain [13]. In parallel, mean values of sacropelvic balance and morphology in healthy subjects of different ages have been published, providing a useful and widespread reference database [4]. 
Compared to the amount of data regarding spinal deformities in patients not affected by neurological disease, scientific papers focusing on sacropelvic morphology and sagittal balance in patients affected by Parkinson's disease (PD) are represented by few case studies [11] or cohorts [7]. Nevertheless, the study of sacropelvic and sagittal balance in this population can be of particular interest because of their intrinsic correlation with postural modulation and the ongoing compensatory mechanisms involving lower limbs [1, 3]. In Parkinson's disease, the surgical correction of spinal deformities is featured by a high risk of short- and long-term complications [7]. As recently shown by Koller et al [7] post-surgical follow-up of PD spinal surgery presents medical and surgical complications, respectively, in 22 and $52 \%$ of the cases, while $33 \%$ of the patients have the possibility to receive further surgical procedures. According to these data, the achievement of a better understanding of pathological adaptation in PD patients' spine would be of interest in the prospective view of improving the conservative management including orthosis, exercise and behavioral treatments. It is indeed well known that the pelvis shape has a straight influence on the pelvic incidence (PI) value, and consequently on the sacral slope (SS): this is directly linked to the shape and degree of Lumbar lordosis (LL) and thoracic kyphosis (TK) in the human spine $[6,15]$. Given these biomechanical correlations, the mechanical forces bearing on the spine and the intervertebral disc units are in some way regulated by SS and PI, in a dynamic relationship that changes according to a multifactorial phenomenon that has to be carefully considered in examining spinal deformities of PD patients.

Since consistent data concerning the relationships between sacropelvic parameters, sagittal balance and postural instability in patients with PD are not available from the literature, the aim of the current study is to describe and compare the disease-related sagittal balance changes with the sacropelvic morphology in a cohort of 31 adults affected by PD, showing different levels of postural instability and duration of the disease (years from diagnosis).

\section{Methods}

\section{Design}

This was a cross-sectional study.

\section{Participants}

Thirty-one consecutive community-dwelling patients (26 males, 5 females) who were diagnosed with idiopathic PD by a neurologist according to the UK Brain Bank Parkinson's criteria have been included in the study protocol [16]. They were enrolled consecutively during a medical examination in a rehabilitation department and were aged 55-83 years (mean $69.5 \pm 7.9$ years, BMI $27.7 \pm 7.7 \mathrm{~kg} / \mathrm{m}^{2}$ ). None of the participants exhibited dyskinesias. All participants were independent with ambulation, in case with the use of assistive devices. All participants followed their normal medication regimen during testing and the functional evaluation was performed during the $\mathrm{ON}$ phase. Participants were excluded if they had parkinsonian disorders as progressive supranuclear palsy, Shy-Drager syndrome, corticobasal degeneration, secondary parkinsonism, familial parkinsonism, a history of dementia as reported by the family or caregiver or if they were unable to stand unassisted for $5 \mathrm{~min}$ without an assistive device. In addition, the participants' ability to follow simple instructions, as determined by their responses to questions and instructions, was assessed during the medical visit and consent process. Informed consent was received from each participant.

\section{Clinical measurements}

Within a multidimensional evaluation model, the Hoehn and Yahr scale (range 0-5) has been used to define the degree of impairment [17, 18]. The Tinetti Scale (range $0-28$, cut-off value for high risk of falling $<19$ points) has been used to detect the risk of falling and to study the differences in sacropelvic morphology. This functional test has been previously proposed in the literature as a reliable measure of dynamic balance in patients affected by PD $[19,20]$.

Clinical examination included body weight, standing height, body mass index, plumb line distance in millimeters from C7, kyphosis apex, L3 and S1 [26-28].

All the functional scores have been collected by the same evaluator.

\section{Radiographic measurements}

Lateral and frontal spine radiographs were taken for each patient using a standardized procedure in the same radiological center. A $230 \mathrm{~cm}$ distance was maintained between the subject and the radiographic source. Each patient maintained the same comfortable standing position with the upper limbs raised forward and fists placed on the clavicles as described by Horton et al [21]. For ethical reasons, the radiographic sessions were performed only during the ON phase of the therapy. With this protocol, T12, the lumbar spine, the sacrum and both femoral heads were visible for the subsequent radiographic evaluation. All radiological measures were performed by the same operator. 
As described by the literature [1], the spinal parameters evaluated in this research were: lumbar lordosis (LL); thoracic kyphosis (TK); spinosacral angle (SSA); spinopelvic angle (SPA); Spinal tilt (ST) (Table 1).

The evaluation of pelvic parameters included pelvic incidence (PI), sacral slope (SS) and pelvic tilt (PT) (Fig. 1). As described by Mac-Thiong et al [5] the relationships between the parameters of sacropelvic balance and morphology have been studied calculating the following ratios SS to PI (SS/PI), PT to PI (PT/PI), PT to SS (PT/SS) (Table 1). This set-up of radiographic parameters has been used to stage the evolution of spinal imbalance severity in different patients (Fig. 2).

\section{Statistics}

The data have been analyzed using the SPSS 3.0 software (SPSS Inc., Chicago, IL, USA). Descriptive statistics (mean and standard deviation) were provided for all subjects, as well as for males and females, respectively. The relationships between each spinopelvic parameter and age, years from diagnosis, Tinetti score were assessed using Pearson's coefficients. Statistically significant correlation coefficients were considered clinically large if above 0.5 , moderate between 0.49 and 0.3 , and small between 0.29 and 0.1 [22]. A level of significance at 0.05 was used for the statistical analyses.

Table 1 Main radiographic parameters described in the study

\begin{tabular}{|c|c|}
\hline Parameter & Definition \\
\hline Kyphosis (TK) & Angle between $\mathrm{T} 12$ and $\mathrm{T} 4$ \\
\hline Lordosis (LL) & Angle between S1 and L1 \\
\hline $\begin{array}{l}\text { Pelvic incidence } \\
\text { (PI) }\end{array}$ & $\begin{array}{l}\text { Angle described by a line perpendicular to S1 } \\
\text { endplate and one drawn from S1 endplate to the } \\
\text { center of the femoral head }\end{array}$ \\
\hline Pelvic tilt (PT) & $\begin{array}{l}\text { Angle described by a line perpendicular the } \\
\text { center of femoral head and the one drawn from } \\
\text { S1 endplate to that point }\end{array}$ \\
\hline Sacral slope (SS) & $\begin{array}{l}\text { The angle between the horizontal and the sacral } \\
\text { plate }\end{array}$ \\
\hline $\begin{array}{l}\text { Spinosacral angle } \\
\text { (SSA) }\end{array}$ & $\begin{array}{l}\text { The angle between the sacral plate and a line } \\
\text { running from the center of } \mathrm{C} 7 \text { to the center of } \\
\text { the sacral plate }\end{array}$ \\
\hline $\begin{array}{l}\text { Spinopelvic } \\
\text { angle (SPA) }\end{array}$ & $\begin{array}{l}\text { The angle between the line drawn from the } \\
\text { femoral head center to sacral plate and a line } \\
\text { running from the center of } \mathrm{C} 7 \text { to the center of } \\
\text { the sacral plate }\end{array}$ \\
\hline Spinal tilt (ST) & $\begin{array}{l}\text { Angle subtended by the horizontal line and the } \\
\text { line from the center of } \mathrm{C} 7 \text { vertebral body to the } \\
\text { center of the upper sacral endplate }\end{array}$ \\
\hline $\mathrm{PT} / \mathrm{PI}$ & Ratio between pelvic tilt and pelvic incidence \\
\hline $\mathrm{SS} / \mathrm{PI}$ & Ratio between sacral slope and pelvic incidence \\
\hline PT/SS & Ratio between pelvic tilt and sacral slope \\
\hline
\end{tabular}

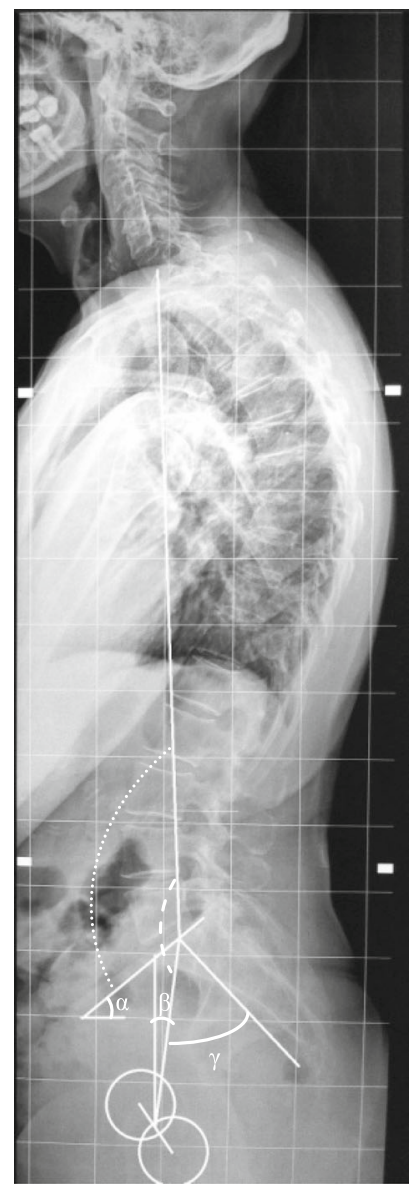

Fig. 1 Main parameters of spinal alignment measured in the study: dotted line spinopelvic angle (SPA), broken line spinosacral angle (SSA), $\alpha$ sacral slope, $\beta$ pelvic tilt, $\gamma$ pelvic incidence

\section{Results}

Clinical characteristics of the participants

Descriptive statistics for demographics, pain and functional parameters including mean values of Hoehn and Yahr score, Tinetti score are presented in Table 2. According to Hoehn and Yahr classification, the participants were staged as belonging to Class 1 in $9.7 \%$ of the cases, 1.5 in $6.5 \%$, 2 in $38.7 \%, 2.5$ in $25.8 \%$ and 3 in $19.4 \%$. Nineteen $(61.2 \%)$ patients exhibited bilateral PD involvement, with minimal to moderate balance impairment. Seven patients $(22.5 \%)$ had at least one accidental fall during the 6 months before the medical examination, and 5 (16.1\%) were presenting a camptocormic behavior while walking; 21 patients $(67.7 \%)$ had an anterior decompensation (average distance from plumb line and kyphosis apex $32.6 \pm 27.7 \mathrm{~mm})$ while $10(32.3 \%)$ had a posterior one (average distance of plumb line from S1 $24.5 \pm 9.4 \mathrm{~mm}$ ); 8 patients $(25.8 \%)$ had a scoliosis above $20^{\circ}$ (Cobb angle $\left.=30.8 \pm 14.4^{\circ}\right)$, while $11(35.4 \%)$ presented a 

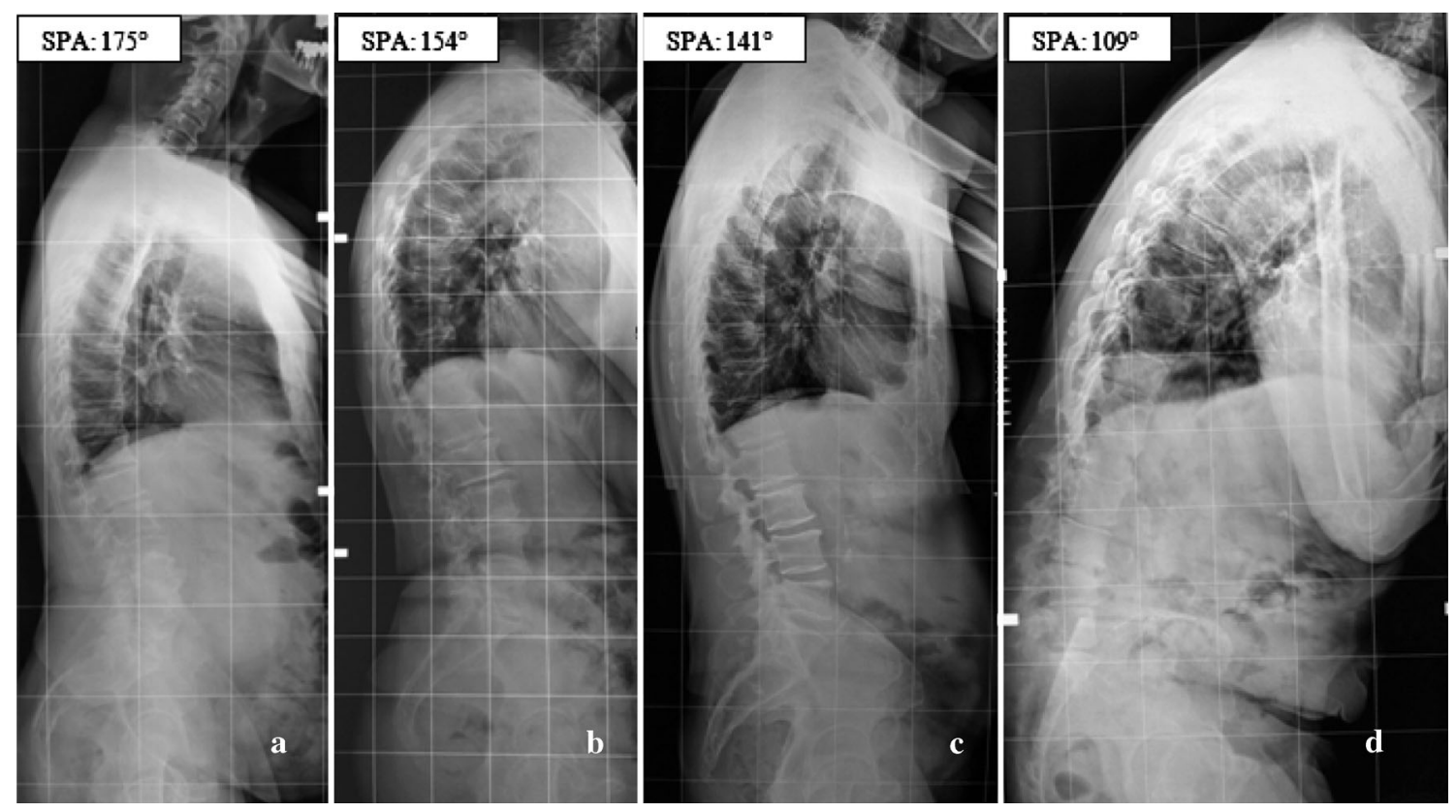

Fig. 2 a-d Variations of spinal alignment in four different PD patients affected by progressive spinopelvic angle (SPA) reduction and anterior imbalance of the trunk

Table 2 Descriptive statistics and functional scores of the cohort

\begin{tabular}{lcc}
\hline Parameter & All $(n=31)$ & Range \\
\hline Age (years) & $69.5 \pm 7.9$ & $50-83$ \\
Height $(\mathrm{cm})$ & $167.6 \pm 7.0$ & $150-181$ \\
Weight $(\mathrm{cm})$ & $64.1 \pm 15.6$ & $50-112$ \\
BMI $\left(\mathrm{kg} / \mathrm{m}^{2}\right)$ & $26.4 \pm 4.6$ & $20-39$ \\
YFD (years) & $7.5 \pm 4.1$ & $0.5-18$ \\
Hoehn Yahr & $2.2 \pm 0.6$ & $1-3$ \\
Tinetti score & $22.1 \pm 5.3$ & $14-28$ \\
\hline
\end{tabular}

$B M I$ body mass index, $Y F D$ years from diagnosis

mild form between $11^{\circ}$ and $20^{\circ}$ (Cobb angle $=$ $\left.11.2 \pm 2.1^{\circ}\right)$.

Sagittal balance radiographic parameters

Even if the average value of radiographic parameters appeared normal (Table 3), 3 patients $(9.6 \%)$ had a LL below $20^{\circ}$, and $3(9.6 \%)$ a lumbar kyphosis; $12(38.7 \%)$ presented a TK above $55^{\circ}$. With regard to the other sagittal parameters considered in the study, pathological values have not yet been defined at present; nevertheless, when compared to healthy adult cohorts reported in the literature $[4,5,13,24]$, although the anatomical parameter PI is similar, the functional PT appears increased and SS decreased. The suggested normality value of 0.5 for the PT/ PI ratio $[1,10]$ is overcome by five patients $(16.1 \%)$. Finally, SSA and SPA are greatly reduced, and ST increased, when compared to healthy individuals.
Table 3 Mean and standard deviation of sagittal sacropelvic parameters of balance and morphology

\begin{tabular}{lcl}
\hline Parameter & All $(n=31)$ & Range \\
\hline Kyphosis $\left(^{\circ}\right)$ & $46.2 \pm 14.5$ & 25 to 76 \\
Lordosis $\left(^{\circ}\right)$ & $44.1 \pm 23.2$ & -18 to 80 \\
Pelvic incidence $\left(^{\circ}\right)$ & $55.1 \pm 13.5$ & 26 to 85 \\
Pelvic tilt $\left(^{\circ}\right)$ & $21.2 \pm 12.1$ & 3 to 52 \\
Sacral slope $\left(^{\circ}\right)$ & $33.8 \pm 10.9$ & 5 to 50 \\
SSA $\left(^{\circ}\right)$ & $108.8 \pm 20.2$ & 39 to 135 \\
SPA $\left(^{\circ}\right)$ & $150.3 \pm 17.1$ & 108 to 178 \\
Spinal tilt $\left(^{\circ}\right)$ & $24.8 \pm 11.1$ & 2 to 50 \\
PT/PI & $0.38 \pm 0.18$ & 0.1 to 0.9 \\
SS/PI & $0.62 \pm 0.19$ & 0.1 to 0.9 \\
PT/SS & $1.02 \pm 1.8$ & 0.1 to 8.6 \\
\hline
\end{tabular}

SSA spinosacral angle, SPA spinosacral angle, $P T / P I$ pelvic tilt/pelvic incidence ratio, SS/PI sacral slope/pelvic incidence ratio, $P T / S S$ pelvic tilt/sacral slope ratio

Radiographic spinal balance and the pelvis

LL is apparently a key curve in this population: indeed, although it is positively correlated with TK $(r=0.50)$, it is mainly positively correlated with the spinal sagittal overall balance described by SSA, SPA and ST ( $r=0.48,0.63$, and 0.56 ); with regard to the pelvis, LL is correlated only with SS $(r=0.53)$. (Table 4).

Apart from the expected correlations between the pelvic parameters, it was found that ST is positively correlated with PI and SS ( $r=0.44$ and 0.47 , respectively), while 
Table 4 Correlation coefficients between sacropelvic parameters and sagittal balance

\begin{tabular}{|c|c|c|c|c|c|c|c|c|c|c|c|}
\hline Parameter & LL & PI & PT & SS & SSA & SPA & ST & $\mathrm{C} 7$ & KA & L3 & $\mathrm{S} 2$ \\
\hline TK & $0.50^{\circ}$ & -0.04 & -0.23 & 0.20 & 0.17 & 0.21 & 0.003 & -0.05 & $-0.46^{\circ}$ & -0.20 & 0.02 \\
\hline LL & & 0.19 & -0.28 & $0.53^{\circ}$ & $0.48^{\circ}$ & $0.63^{\circ}$ & $0.56^{\circ}$ & $-0.52^{\circ}$ & $-0.62^{\circ}$ & 0.32 & 0.27 \\
\hline PI & & & $0.62^{\circ}$ & $0.54^{\circ}$ & -0.07 & -0.20 & $0.44^{\circ}$ & 0.02 & 0.04 & 0.03 & -0.27 \\
\hline $\mathrm{PT}$ & & & & -0.19 & -0.30 & $-0.62^{\circ}$ & 0.04 & 0.20 & 0.26 & 0.17 & -0.32 \\
\hline SS & & & & & 0.22 & $0.42^{\circ}$ & $0.47^{\circ}$ & -0.20 & 0.28 & 0.20 & 0.02 \\
\hline SSA & & & & & & 0.33 & 0.27 & $-0.44^{\circ}$ & -0.37 & 0.24 & 0.27 \\
\hline SPA & & & & & & & 0.30 & $-0.60^{\circ}$ & $-0.60^{\circ}$ & $0.38^{\circ}$ & $0.46^{\circ}$ \\
\hline ST & & & & & & & & -0.32 & -0.24 & 0.38 & 0.05 \\
\hline
\end{tabular}

$T K$ thoracic kyphosis, $L L$ lumbar lordosis, $P I$ pelvic incidence, $P T$ pelvic tilt, $S S$ sacral slope, $S S A$ spinosacral angle, SPA spinosacral angle, $S T$ spinal tilt, $C /$ distance from plumb line and C7, $K A$ apex of TK, $L L$ L3 distance from plumb line, $S 2$ sacral distance from plumb line

${ }^{\circ}$ Statistically significant correlation coefficient $(p<0.05)$

SPA is negatively correlated with PT $(r=-0.62)$, and positively correlated with SS $(r=0.42)$ (Table 4$)$.

Clinical and radiographic measurements of spinal balance

Clinical parameters were mainly negatively correlated with the spinal radiographic parameters: LL with the distance of plumb line from $\mathrm{C} 7$ spinous process $(r=-0.52)$ and the one from plumb line and kyphosis apex $(r=-0.62)$, while TK only with this last one $(r=-0.46)$ (Table 3). Negative correlations have been observed also for SSA with PC7 $(r=-0.44)$, and SPA with the distance of plumb line from $\mathrm{C} 7$ spinous process and the one from plumb line and kyphosis apex (both $r=-060$ ); only for this last parameter some positive correlations were found, with the distance of plumb line to L3 and S1 ( $r=0.38$ and 0.46 ) (Table 4).

\section{Other clinical parameters}

The duration of disease (years from diagnosis) was negatively correlated with the overall sagittal spinal balance, as described radiographically by SSA and SPA $(r=-0.44$ and -0.40 , respectively), and positively with the clinical parameter represented by the distance from plumb line and kyphosis apex $(r=0.36)$. The sagittal balance of the spine on the pelvis, as described by the SPA and ST, was moderately correlated to Tinetti score $(r=0.37$ and 0.48 , respectively) (Table 5).

\section{Discussion}

Given the high risk of short-term and long-term complications after surgical procedures [7], the current research presents the analysis of spinopelvic alignment targeted to
Table 5 Correlation coefficients between sacropelvic parameters and years from diagnosis

\begin{tabular}{lrrrr}
\hline Parameter & YFD & \multicolumn{1}{l}{ Age } & \multicolumn{1}{l}{ BMI } & TINETTI \\
\hline Kyphosis & -0.24 & -0.05 & 0.15 & -0.08 \\
Lordosis & -0.27 & -0.15 & 0.03 & 0.32 \\
Pelvic incidence & 0.05 & -0.08 & 0.06 & 0.12 \\
Pelvic tilt & 0.18 & -0.01 & -0.07 & -0.02 \\
Sacral slope & -0.12 & -0.06 & 0.15 & 0.16 \\
SSA & $-0.44^{*}$ & -0.25 & $-0.43^{*}$ & 0.33 \\
SPA & $-0.40^{*}$ & -0.17 & 0.12 & $0.37^{*}$ \\
Spinal tilt & -0.05 & -0.31 & -0.01 & $0.48^{*}$ \\
PT/PI & 0.15 & -0.02 & -0.08 & -0.09 \\
SS/PI & -0.20 & 0.05 & 0.06 & 0.08 \\
PT/SS & 0.20 & -0.03 & -0.18 & 0.09 \\
\hline
\end{tabular}

$Y F D$ years from diagnosis, $B M I$ body mass index

* Statistically significant correlation coefficient $(p<0.05)$

improve rehabilitation strategies and conservative management of typical trunk neurogenic deformities detected in patients affected by PD. In fact, as known by the literature the risk for pseudoarthrosis in this population can be higher than in general population and the importance of considering these pelvic parameters to help reduce the risk of additional surgeries in this patient group has to be specifically considered before surgical time.

Sagittal balance parameter correlations and clinical consequences

For this cohort of patients affected by PD, some correlations among the overall sagittal balance, as described by SSA and SPA, and the duration of disease have been noted (Table 5). In particular way, these two parameters, but not TK, presented a moderate negative correlation with the years from the diagnosis. 
Furthermore, in contrast with previously reported data $[5,24]$, the lack of correlation with age indicates that the modification of these sagittal balance parameters likely represents a typical pathological adaptation of the spine to the disease. The positive correlation existing among LL, SSA, SPA and ST indicates, anyway, that the preservation of LL is of paramount importance to avoid a progressive sagittal imbalance and pain [10, 12, 14, 23, 24], as seen in the aging process [24] and in other kind of spinal diseases [2, 3, 6, 12, 13]. Under the clinical point of view, these results confirm that, in PD patients, the preservation of LL can be addressed as one of the postural strategies to be soon included in conservative programs after the diagnosis within the aim of preventing the pathological evolution of the SPA and SSA. Ergonomics, education, exercise and orthotics selection should be always pointed to the preservation of LL and SS to prevent a pathological increase of pelvic retroversion (pelvic tilt increase).

Differently to what is typically seen in the literature [13], TK preserves a significant correlation only with LL but not with pelvic parameters such as PI or SS. On the other hand, the positive correlation that these two latter parameters have with ST indicates that PD patients presenting the lowest values of PI have a greater risk of a forward sagittal imbalance and, probably, a greater risk of evolutive spinal deformities on the sagittal plane. According to this consideration, in this subgroup of PD patients a specific preventive rehabilitation program has to be sooner proposed to reduce the possibility of a rapid evolution. Moreover, in functional terms, a moderate correlation between ST and SPA with the Tinetti score was noted, suggesting that the two mentioned parameters can potentially be considered as the main radiological spinal indicators of the risk of falling (Table 5). Thus, it is in our opinion that the prevention of forward spinal imbalance can be seen as a relevant contribution to fall risk reduction in PD patients.

\section{Absolute values of sagittal balance parameters and their clinical consequences}

The reported results constitute a preliminary database that could enable clinicians to perform a better clustering analysis of PD patients according to the different stages of disease or to compare the effects of different rehabilitation strategies or pharmacological approaches on trunk alignment. In particular, the average values derived by the indirect morphological evaluation provided useful preliminary information about the spinopelvic values in PD patients with a history of disease between 6 months and 18 years (mean time $7.5 \pm 4.1$ years) (Table 2 ).

In the studied cohort of PD patients, PI, PT and SS presented values between $26^{\circ}$ and $85^{\circ}, 3^{\circ}$ and $52^{\circ}$ and between $5^{\circ}$ and $50^{\circ}$, respectively (Table 3 ). When considering normal adults, scientific literature report values between $32^{\circ}$ and $74^{\circ}$ for PI, between $0^{\circ}$ and $27^{\circ}$ for PT, and between $24^{\circ}$ and $55^{\circ}$ for SS [4]. Similar ranges for PI $\left(42-88^{\circ}\right), \operatorname{PT}\left(21-50^{\circ}\right)$ and $\operatorname{SS}\left(8-57^{\circ}\right)$ were found in another study conducted on $23 \mathrm{PD}$ patients [7]. Unlike healthy subjects and patients affected by mechanical low back pain or idiopathic scoliosis, a lack of correlation between LL and PI is noticeable (Table 3) [3, 6, 9, 12]. In the group of PD patients we studied, the findings reveal that $\mathrm{SS}$ is the most important and main spinopelvic regulator of LL and that the ability to avoid pelvic retroversion is the way to maintain a correct LL value. The ability to preserve the LL value inside a range of normality is even more important considering the presence of a significantly moderate and positive correlation between LL value and SSA, SPA and ST (Table 3). According to the fact that SPA and ST values presented a positive correlation with Tinetti score, it can be argued that preservation of LL is of paramount importance in determining the global sagittal alignment of the spine in PD patients and consequently the reduction of fall risk due to sagittal imbalance [10].

Pelvic parameter variations and their clinical consequences

The analysis focused on the morphological and radiographic parameters that are most commonly used by clinicians to evaluate the sagittal sacropelvic balance and morphology in the presence of spinal deformity. We know from the scientific literature that for healthy individuals the PT value is less than half of the PI value, while the SS is more than half the value of PI $[1,4,5]$. In the studied group of PD patients, PT/PI ratio and SS/PI are on the average $<0.5$ and over 0.5 , respectively, which is in agreement with previously reported data [4]. A significant set of patients $(32.2 \%)$ overcame these normality boundaries. In PD patients as well, values outside those ranges could potentially predispose to the development of spinal pathology, but further studies are needed to evaluate the biomechanical and clinical consequences in this specific population. PT and SS values can help to detect early compensation from the pelvis (increased pelvic retroversion with sacral verticalization) in the context of spinal pathology, as often found in spondylolisthesis [1, 9] or global sagittal spinal imbalance in degenerative diseases of the spine [10, 12]. As typically seen in these spinal pathologies, in the studied group of PD patient, PT and SS presented an increasing and decreasing tendency, respectively. These findings confirm that the increased retroversion of the pelvis represents a compensation to the mechanical degenerative processes occurring in the aging spine and to the related decrease of LL that induces a 
positive spinal balance during the aging process [24]. Given the fact that changes in PT and SS with age are of minor importance in healthy individuals, a relevant increase in PT and/or decrease in SS has to be considered as a potential factor for further evolution in neurogenic spinal deformity.

Overall sagittal balance parameters and their clinical consequences

The reduction of SSA appreciated in this cohort corresponded to $83 \%$ of the reference value reported in the literature [4] (normality range $130.4 \pm 8.1^{\circ}$; our results $108 \pm 20.2^{\circ}$ ). This pronounced alteration can be considered as a specific pathological adaptation to PD in light of the fact that patients affected by non-neurogenic diseases or by mechanical diseases of the spine, such as degenerative disc disease or spondylolisthesis, are usually presenting larger values of SSA [10, 12, 14]. In particular, as already noted by other authors [23], SSA and SPA reductions can be considered as morphological adaptations that are specifically linked to the duration of the disease. In agreement with biomechanics considerations arising from the literature, the ST reduction can be considered as the main radiographic parameter related to the risk of falling in PD patients (see Table 5) [25]. Among the different morphological parameters included in the physical examination, the distance from plumb line to the bony marker of $\mathrm{C} 7$ presented a negative correlation with SPA (Table 4). Consequently, this parameter can be considered as the main morphological indicator of positive sagittal imbalance during a routine medical examination to screen an eventual progression in spinal deformity observed in PD patients.

\section{Limits and strengths}

The main limitation of the study is represented by the limited number of participants and the absence of a control group of healthy subjects or not affected by a neurogenic deformity. However, we aimed to propose some preliminary considerations based upon our data and to pose the basis for further analyses in larger groups of patients affected by PD. For ethical reasons, we considered to avoid the inclusion of healthy subjects because the existing literature is providing a reliable and large database about sagittal balance parameters in healthy subjects of similar age.

\section{Conclusions}

Looking at the pelvis is opening new important insights in spinal disease management, particularly where surgery is concerned, and novel applications in rehabilitation management are constantly underway. Until now, only one study faced the issue of radiographic sagittal balance in Parkinson's disease focusing chiefly on highly compromised patients who had fusion. In our study, instead, we evaluated a broader and more heterogeneous population of PD patients with the intent to have a more representative clinical sample. Parameters like SSA, SPA, ST, as well as SS/PI and PT/PI ratios, may have relevant significance in terms of functional implications at the spinal level and the related fall risk in PD patients, suggesting that further research on larger cohorts of subjects may be worthwhile.

Acknowledgments The study has been fully conducted in the Rehabilitation Service of Fondazione Teresa Camplani-Domus Salutis Rehabilitation Hospital, Brescia, Italy.

Conflict of interest The authors of the paper have not received specific funding to perform the research. The authors declare no conflict of interest. Luciano Bissolotti: employed at the Fondazione Teresa Camplani-Domus Salutis Rehabilitation Hospital, Brescia. Massimiliano Gobbo: employed at the Department of Clinical and Experimental Sciences, University of Brescia, Brescia, Italy. Jorge Hugo Villafañe: employed at the IRCCS Fondazione Don Gnocchi ONLUS, Milan, Italy. Stefano Negrini: Professor of Physical and Rehabilitation Medicine, University of Brescia, Italy; IRCCS Fondazione Don Gnocchi ONLUS, Milan, Italy.

\section{References}

1. Roussouly P, Nnadi C (2010) Sagittal plane deformity: an overview of interpretation and management. Eur Spine J 19(11): 1824-1836 (Epub 2010 Jun 22)

2. Labelle H, Roussouly P, Berthonnaud E, Dimnet J, O'Brien M (2005) The importance of spino-pelvic balance in L5-S1 developmental spondylolisthesis: a review of pertinent radiologic measurements. Spine (Phila Pa 1976) 30(6 Suppl):S27-S34

3. Obeid I, Hauger O, Aunoble S, Bourghli A, Pellet N, Vital JM (2011) Global analysis of sagittal spinal alignment in major deformities: correlation between lack of lumbar lordosis and flexion of the knee. Eur Spine J 20(Suppl 5):681-685

4. Mac-Thiong JM, Roussouly P, Berthonnaud E, Guigui P (2010) Sagittal parameters of global spinal balance: normative values from a prospective cohort of seven hundred nine Caucasian asymptomatic adults. Spine (Phila Pa 1976) 35(22):E1193-E1198

5. Mac-Thiong JM, Roussouly P, Berthonnaud E, Guigui P (2011) Age- and sex-related variations in sagittal sacropelvic morphology and balance in asymptomatic adults. Eur Spine J 20(Suppl 5):S572-S577

6. Roussouly P, Pinheiro-Franco JL (2011) Biomechanical analysis of the spino-pelvic organization and adaptation in pathology. Eur Spine J 20(Suppl 5):S609-S618

7. Koller H, Acosta F, Zenner J, Ferraris L, Hitzl W, Meier O, Ondra S, Koski T, Schmidt R (2010) Spinal surgery in patients with Parkinson's disease: experiences with the challenges posed by sagittal imbalance and the Parkinson's spine. Spine J 19(10): 1785-1794

8. Mac-Thiong J-M, Labelle H, Charlebois M (2003) Sagittal plane analysis of the spine and pelvis in adolescent idiopathic scoliosis according to the coronal curve type. Spine 28:1404-1409 
9. Tanguay F, Mac-Thiong J-M, de Guise JA (2007) Relation between the sagittal pelvic and lumbar spine geometries following surgical correction of adolescent idiopathic scoliosis. Eur Spine J 16:531-536

10. Le Huec JC, Charosky S, Barrey C, Rigal J, Aunoble S (2011) Sagittal imbalance cascade for simple degenerative spine and consequences: algorithm of decision for appropriate treatment. Eur Spine J 20(Suppl 5):S699-S703

11. Upadhyaya CD, Starr PA, Mummaneni PV (2010) Spinal deformity and Parkinson disease: a treatment algorithm. Neurosurg Focus 28(3):E5

12. Barrey C, Roussouly P, Perrin G, Le Huec JC (2011) Sagittal balance disorders in severe degenerative spine. Can we identify the compensatory mechanisms? Eur Spine J 20(Suppl 5):626-633

13. Chaléat-Valayer E, Mac-Thiong JM, Paquet J, Berthonnaud E, Siani F, Roussouly P (2011) Sagittal spino-pelvic alignment in chronic low back pain. Eur Spine J 20(Suppl 5):634-640

14. Schuller S, Charles YP, Steib JP (2011) Sagittal spinopelvic alignment and body mass index in patients with degenerative spondylolisthesis. Eur Spine J 20(5):713-719

15. Stagnara P, DeMauroy JC, Dran G, Gonon G, Costanzo G, Dimnet J, Pasquet A (1982) Reciprocal angulation of vertebral bodies in a sagittal plane: approach to references for the evaluation of kyphosis and lordosis. Spine (Phila Pa 1976) 7(4):335-342

16. Hughes AJ, Daniel SE, Kilford L, Lees AJ (1992) Accuracy of clinical diagnosis of idiopathic Parkinson's disease. A clinicopathological study of 100 cases. JNNP 55:181-184

17. Hoehn MM, Yahr MD (1967) Parkinsonism: onset, progression and mortality. Neurology 17:427-442

18. Scalzo PL, Flores CR, Marques JR, Robini SC, Teixeira AL (2012) Impact of changes in balance and walking capacity on the quality of life in patients with Parkinson's disease. Arq Neuropsiquiatr 70(2):119-124
19. Kegelmeyer DA, Kloos AD, Thomas KM, Kostyk SK (2007) Reliability and validity of the Tinetti Mobility Test for individuals with Parkinson disease. Phys Ther 87(10):1369-1378

20. Brusse KJ, Zimdars S, Zalewski KR, Steffen TM (2005) Testing functional performance in people with Parkinson disease. Phys Ther 85:134-141

21. Horton WC, Brown CW, Bridwell KH (2005) The effect of arm position on sagittal plane alignment. Spine 30:427-433

22. Cohen J (1998) Statistical power analysis for the behavioural sciences, 2nd edn. Lawrence Erlbaum Associates, Hillsdale, pp 100-110

23. Kashihara K, Imamura T (2012) Clinical correlates of anterior and lateral flexion of the thoracolumbar spine and dropped head in patients with Parkinson's disease. Parkinsonism Relat Disord 18(3):290-293

24. Hammerberg EM, Wood KB (2003) Sagittal profile of the elderly. J Spinal Disord 16:44-50

25. Le Huec JC, Saddiki R, Franke J, Rigal J, Aunoble S (2011) Equilibrium of the human body and the gravity line: the basics. Eur Spine J Suppl 5:558-63

26. McLean IP, Gillan MG, Ross JC, Aspden RM, Porter RW (1996) A comparison of methods for measuring trunk list. A simple plumbline is the best. Spine 21(14):1667-1670

27. Zaina F, Atanasio S, Ferraro C, Fusco C, Negrini A, Romano M, Negrini S (2009) Review of rehabilitation and orthopedic conservative approach to sagittal plane diseases during growth: hyperkyphosis, junctional kyphosis, and Scheuermann disease. Eur J Phys Rehabil Med 45(4):595-603

28. Negrini S (2009) Focus on flexed posture and hyperkyphosis: prevention and rehabilitation to reduce disability and increase quality of life. Eur J Phys Rehabil Med 45(4):567-569 